\title{
Aspirin discontinuation increases risk of cardiovascular events
}

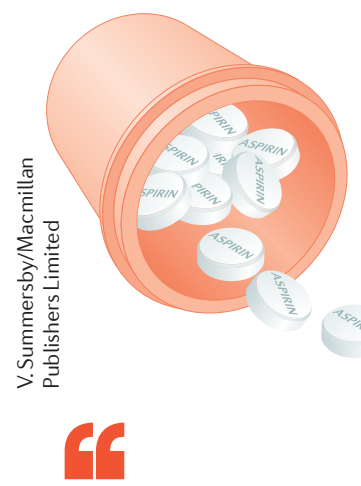

Patients who

persisted with

aspirin therapy

throughout

the follow-up

period had

the lowest

incidence of

cardiovascular

events

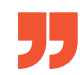

Many long-term users of low-dose aspirin consider stopping their treatment, but the risks associated with aspirin discontinuation unrelated to surgery or bleeding events have not been known. In a Swedish study published in Circulation, Johan

Sundström and colleagues report that patients who discontinue aspirin have a $37 \%$ higher rate of cardiovascular events compared with those who continue therapy.

"Previous experimental studies have suggested a possible rebound effect after aspirin discontinuation," comments Sundström. "Conversely, an acutely increased risk of cardiovascular events after aspirin discontinuation has been observed in several studies, but the reasons for aspirin withdrawal in those studies were related to surgery or bleeding." The study investigators sought to determine the risks of aspirin discontinuation in a large, nationwide cohort of patients who recieved long-term aspirin therapy for primary and secondary prevention.

Individuals aged $>40$ years in the Swedish prescription register who were prescribed low-dose aspirin were enrolled in the study. Given that the study investigators wanted to assess the effect of treatment breaks and permanent discontinuation after a 1-year period of continuous aspirin treatment, only patients who demonstrated $\geq 80 \%$ adherence to therapy were included.

In total, 601,527 patients taking low-dose aspirin were included in the study and followed up for a median of 3 years. Approximately $15 \%$ of the cohort discontinued aspirin treatment after this period. Patients who persisted with aspirin therapy throughout the follow-up period had the lowest incidence of cardiovascular events, whereas those who discontinued aspirin had a $37 \%$ higher rate of cardiovascular events. This higher rate corresponded to one additional cardiovascular event observed per year in 1 of every 74 patients who stopped aspirin therapy. Moreover, the risk seemed to increase as soon as patients discontinued aspirin, and was even further elevated in those of older age and with previous cardiovascular disease. Among the $46 \%$ of patients who were likely to be receiving aspirin for primary prevention, aspirin discontinuation resulted in a $28 \%$ higher rate of cardiovascular events than continuing aspirin.

"Adherence to low-dose aspirin treatment in the absence of major surgery or bleeding may be an important treatment goal," concludes Sundström. "This study may help physicians and patients to make an informed decision on whether or not to stop aspirin use."

Karina Huynh

ORIGINAL ARTICLE Sundström, J. et al. Low-dose aspirin discontinuation and risk of cardiovascular events. Circulation 136, 1183-1192 (2017) 